\title{
Erhard Denninger Neue Rechte im technologischen Zeitalter?
}

$I$.

Man erlaube mir, das Thema "Neue Rechte im technologischen Zeitalcer $\approx$ mit einem Fragezeichen zu versehen. Damit soll nicht ein vorureilsvoller Zweifel an der Exiscenz solcher "neven o oder mneuartigen Rechte angemeldet werden und schon gar nicht an der Tarsache, daß die technologische Entwicklung nicht nur unsere Lebensbedingungen, sondern mit diesen auch unsere rechclichen Ordnungen tiefgreifend umgestaliet. Vielmehr soll das Fragezeichen andeucen, daß dieser Wandel eine Viclzahl unbeantworteter Fragen aufwirft, von denen einige hier näher betrachtet werden sollen. So wissen wir z.B. nichts Genaues über Art und Grad der notwendigen Abhängigkeit des rechelichen Wandels vom technischen Wandel. Zeichnet dieser jenem eine bestimmte Richtung - mit Notwendigkeit? - vor oder kann die Gescllschaft zwischen mehreren Optionen wählen? Welches Maß an Steucrungschancen crgibt sich aus diesem Verhälnnis für das Rechtssystem gegenüber der technologischen Entwicklung - oder behäls Ernst Forstboff mir seiner Prognose recht, daß alle selbständigen geistig-politischen Konzeptionen an den ehernen Sachzwängen der stechnischen Realisacion * scheitern werden?' Oder betrachten wir die Situacion des Individuums: Weiche Qualitär besitzen diese «neuen * Rechre, welche Funktionen erfüllen sie? Sollen sie dem Einzelnen in erster Linie Schurz gewähren oder dienen sie - als woffensivex Rechte - seiner weiteren Ent\{altung, seiner noch intensiveren Herrschaft über Natur und Mirwelt? Wie fügen sie sich rechtslogisch, verfassungstheoretisch und normpraktisch in das überkommene Rechtsbegriffs- und Rechtsschutzsystem ein? Sichern sic dem Einzelnen letzelich das nowwendige $\mathrm{MaB}$ an Frciheit in einer durch und durch verwalteten und technisierten Wele oder nicht? Fragen über Fragen, für die mein kleines Fragczeichen am Antang als Chiffre stehc.

II.

1. Versuchen wir zunächse, genauer zu fassen, wovon die Rede sein soll. Wenn wir feststellen, daß sich unter der Überschrift wNeve Rechte des technologischen Zeitalcers" gar kcinc fest abgegrenzte, homogene Gruppe von Rechtsphänomenen versammelt, sondern höchst unterschiedliche Erscheinungen, dann schärft sich unser Blick für dic Norwendigkeit, dic Ausgangsfragen schr differenziert anzuge- 
hen. Der eine denkt zuerst an die Chancen, auch an die ökonomischen Chancen gentechnologischer Experimence und Auswercungen, der andere an ihre Gefahren, zumal im Bereich der Humangenetik. Ein dritter denkt an die Verwertung dee Kernenergic; ein vierter an sein Recht, private Fernsehprogramme auszustralalen und zu verkaufen; ein fünfter möchte Manganknollen und andere kostbare Erze vom Grunde der Tressee „fischen ; $^{2}$ und ein sechster begchrt schlicht den Schutz seiner persönlichen Daten gegen elektronisch automacisierte und damit ubiquitare Behördenneugier. Das Bundesverfassungsgericht hat hieffür den Terminus "Recht auf informationelle Selbstbestimmung « aus der Literatur übernommen ${ }^{3}$ und dieses Recht sozialethisch und demokratietheorerisch uncermaucrt.

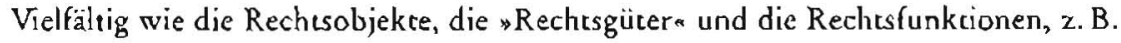
Hcrrschaftssicherung oder Verwertung oder Förderung oder Eingriffsabwehr, sind auch die anerkannten oder um Anerkennung kämplenden Rechtssubjekte, die potentiellen Rechrsinhaber sneuer Rechrea und die ihnen zugeordnercn Pflichrsubjekte. Das Zweierschema Staat-Individuum ist brüchig; längst stehen außerdem Gruppen, "Initiativen «, "Betrolfene", Allgemeinheiten « und ihre Verterer sowie jurisrische Kollektive aller Art auf der Bühne. Die herkömmlich am dualistischen Schema orientierte und an klare juristische Zurechnungsendpunkre, Rechrs- und Haftungssubjekte gewöhnte Verwaltungsrechusdogmarik kämpft einen verzweifciten, wohl hoff́nungslosen Kampf, um der Fülle der Erscheinungen in den neuen, wie es heißr: mehrpoligen * Vervaltungsbezichungen Herr zu werden.

2. Die Komplexitär der Fragestellung wird weiterhin deuclich, wenn man nach Unterschieden in der Entwicklung auf den verschiedenen Ebenen der Rechesgelcung, nach Abscufungen im Universalitütsanspruch internationaler Regelungen fragr. Daß die rasance Encwicklung der modemen Verkehrs- und Kommunikacionstechniken das BewuBtsein für die weltweire Interdependenz nichr nur der politischen und ökonomischen, sondern auch der rechtlichen Beziehungen gebilder hac, ist freilich einc Binsenweisheit. Weniger gemeinplätzig ist die Beobachtung, daß die jüngste Entwicklung des Volkerrechts, die im wesentlichen als Auseinanderserzung um die Vervollkommoung des Systems der individuellen und kollekiven Menschenrechte geführt wird, unmistelbar durcla die tcchnologische Entwicklung angestoßen und inhaltlich bescimmt wird. Ich spreche von der ihrerseits in rascher Veränderung begrilfenen Rechtsmasse, welche man als nMenschenrechte der dritten Generation " zu bezeichnen pflegt und für die Eibe Riedel mit guten Gründen die Kennzeichnung als "Menschenrechte der dritten Dimension « vorschlägt.

Ein Merkmal dieser Rechre wie des Rechts auf eine lebenswerte Umwelt, des Rechis auf Entwicklung, des Rechts auf Frieden, des Rechts auf Teilhabe am gemeinsamen Menschleitserbe, des Rechts auf Selbstbestimmung, Partizipation und Kommunikation ist ihre programmbafte Unbestimmtheit, ihre Nichteignung für den unmitrelbaren adminiscrativen Vollzug. Auf dic Ebene innerstaarlichen Rechis übertragen würde man sich derartige Rechte nur als Staatszielbestimmungen oder Gesetzgebungsaufiräge, kcinesfalls aber als unmitrelbar einklagbare subjcktive Berechrigungen des Einzelnen vorstellen können. In diesem Sinne handelt es sich hier um wwerdendes Recht “, dessen rechts- und allgemein-politische Dynamik, ja Brisanz, abcr nicht unterschätzt werden sollte. In unserem Problemzusammenhang ist bemerkenswert, daß der wissenschaftlich-technologische Fortschritt völkerrechelich

\footnotetext{
z Zu den Rechisfragen: G. Jaenicke/E.Schanize/W. Hauscr, A Jom Venture Agrecment for Senbed Minung, Studies in Transnational Law of Natural Resources, Vol. S, $19 \$ 1$.

3 BVeriGE 65, 1, 43; Nachwese bei [F. Denninger, Das Rechi auf informaetonelle Selbsebesummung und Innere Siclicrhert, KJ $1985,215,218$.

4 E. Ricdel, Munschenrechte der dntun Dimension, EuGRZ 1989 . gff.
} 
als ein universelles, menschbcicliches Phänomen (und Problem) wahrgenommen und verarbeitce wird. Das gilt von seinen Gefahren und Risiken ebenso wie von seinen Reichtums- und Machtentfaltungschancen. Dieses Bewußtsein hat schon 1975 in der "Declaration on the Usc of Scientific and Technological Progress in the Intercsts of Peace and for the Bcnefit of Mankind der Vereinten Nationen Ausdruck gefunden':

"All Statcs shall promote international co-operation to ensurc that the results of scientific and tcchnological devclopments are used in the interests of strengthening international peace and securicy, freadom and independence, and also for the purpose of the economic and social development of peoples and the realization of human rights and frcedoms in accordance with the Charter of the Unied Nationsu.

Die weltweite Erkennenis, daß der technische Fortschritt (der Waffensysteme) den zur Vernichtung einer bestimmten Anzahl von Menschen erforderlichen Aufwand an Zcit und Menschenkraft in der Geschichte stetig verringert hat ${ }^{6}$ und daß heute die totale Selbstausrottung der Menschheit aul dem Spiele steht, hat menschenreclitlich ihren Nicderschlag in der Proklamierung eines Rechtes auf Frieden gefunden. Am 12. November 1984 proklamierte die General Assembly der Vercinten Nationen sfeierlich , wthat the peoples of our planec have a sacred right to peacen und dementsprechend, that the preservation of the righr of peoples to peace and the promotion of its implementation constitute a fundamental obligation of cach State $\alpha$.

Nicht nur die zerstörerischen Kräfte und Möglichkeiten der Technik beschäftigen die Phantasie der Völkerrechtsjuristen, sondern auch ihre Glück und Wohlfahr verheißenden. Diese positiven Seiten stchen als ungeschriebene Voraussetzung hinter der Konzcption eines Rechzes aw Entwrcklung, wie es in der UN-Declaracion on the Right to Development vom 4. Dezember $1986^{3}$ als Recht sowohl jedes Einzelnen als auch jedes Volkes (Art. 1, 1.) vorgestellt wird: Dort ist zwar, in ausdrücklicher Erinnerung an die beiden universellen Menschenrechispakte der Vereinten Nationen vom 19. Dezember 1966, nur von den wirtschaftlichen, sozialen, kulturellen und polirischen Dimensionen der Entwicklung die Rede, doch war und ist jedermann klar, daß deren Entfaltung nur auf der Basis eincr hochentwickelten und noch weiter zu entwickelnden wissenschaftich-technischen Zivilisation möglich ist.

Daran, daß die Fortschritte der Raumfahrttechnik die Herausbildung einer extraterrestrischen Rechtsordnung erzwingen, dic sich um wdie geordnete und sichere Erschließung des Mondes $\alpha$ - so der Mondvertrag von 1979, Arr. 11 (7) - ebenso kümmern muß wie um die Beseirigung des Weltraum-Abfalls, ist hier nur zu erinnern. Dic Bewältigung der Technik-Folgen auf unserem klein und eng gewordenen Planeten bietet rechtliche Probleme genug. ${ }^{10} \mathrm{Zu}$ den umstrittensten gehören die rechtliche Bedeutung und Funktion des Prinzips des "Common Hentage of Mankind "', bei dessen Ervähnung der moderne Jurist weder an die Bhagavadgica noch an die Ancigone des Sophokles oder an Leonardos Mona Lisa zu denken hat,

5 Gereral Asscmbly resolution 3184 (XXX) of to November 1979 .

6 Dazu: H. Popicz. Phanomene der Machi, 1986, S. 126.

7 General Asscmbly resolucion $39 / 11$ of 12 November 1984 .

8 General Assicmbly resoluuon 41/128 of 4 Deecmber 1984 .

9 Texe in: M. Schwerzer/N". Rudolf, Fricdensvolkerrechi, ). Aufl. 1985, Nr. 45, 48g [f. Vgl. Riedel (Fn. 4), 197.

$10 \mathrm{Vgl}$. den Kasalog im Abschlußdokument des WTiener KSZF-Folgetreffens vom IS.Januar I9\$9. Ab. setniex Zusammenarbeil in den Bireichen der Wirschall, der Wissenschafe und der Technik sowse der Umwelt, Nr. 24 KL, Texe in: EuGRZ 1989, 85,90 f

II Vgl dazu Riedal (Fn. 4), is ff. 
sondern an die Ausbeutung des Mceresgrundes. Art. 136 der UN-Seerechtsübereinkunft von $1982^{12}$ statuiert: "The Area and its resources are the common heritage of mankind. Hinter dem hier bekundeten Menschheits-Pathos verbirgt sich die Gerechrigkeits- und Solidaricätsforderung der Entwicklungsländer im Nord-SüdDialog, dic befürchten, wegen fehlenden Kapitals und fehlenden rechnischen Knowhow's von der Nutzung der Tiefsee-Bodenschätze ausgeschlossen zu bleiben. An diesem nur scheinbar etwas abgelegenen, wcil aul dem Grunde des Ozcans angesiedelten Beispiel wird deutlich, daß die rechnologische Entwicklung uns zwingt, die sozıalethischen Grundlagen unserer modernen Rechtssysteme zu überdenken. Die Idec, daß die natürlichen Reichtümer der Erde das "Common heritage of mankind « bilden oder, schärfer und konflikurächtiger formuliert, daß im nNaturzustand " der Menschheic alle ein "Rechr" auf alles haben, war einc der tragenden Säulen der klassischen Sozialphilosophie des 17./18. Jahrhunderts. "Die Erde und alles, was in ihr ist, " sagt John Locke, wist den Menschen zum Unterhalt und zum Genuß ihres Daseins gegeben. Alle Früchte, die sie auf natürliche Weise hervorbringt, und alle Tiere, die sie ernährt, gehören den Menschen gemeinsam, ... und niemand hat über irgend ctwas, so wie es sich in einem natürlichen Zustand befindec, ursprünglich ein privaces Herrschaftsrecht, welches das der übrigen Menschen ausschlösse. " " Und Rousseau sieht in der Eigentums-Behauptung (im doppelten Wortsinn) den eigentlichen Gründungsakt der bürgerlichen Gesellschafr, d.h. der staatlichen Rechtsordnung.

"Der erste, dem es in den Sinn kam, ein Grundstück einzuhegen und zu bchaupten: ,Das gehört mir،, und der Menschen fand, einfältig genug, ilım zu glauben, war der eigentlichc Grinder der bürgerlichen Gesellschaít. Wieviel Verbrechen, Kricge, Mordinten, Elend und Scheußliclzkeic häcte der Mann dem Menschengeschlecht erspart, der die P(ähle herausgerissen, den Graben eingecbnet und seinen Mitmenschen zugerufen hätte: ,Hutet euch, diesem Betrüger zu glauben! Ihr seid verloren, wenn Ihr vergeßt, daß die Früchte allen gehören und die Erde niemandem! ' $^{\text {'4 }}$

Die zweite tragende Säulc nicht nur der Philosophie, sondern vor allem der Praxis der Aufklärung und des ihr folgenden technologischen Zeitalters bis heute ist die Überwindung des potentiellen Ur-Kommunismus durch das konkretisierende und individualisierende Eigentumsrecht und seine Legitimation durch individuelle $\mathrm{Ar}$ beitslerstung. Dies gilt von John Locke bis zum Eigentumsschurz sozialversicherungsrechtlicher Rechispositioncn durch das Bundesverlassungsgericht." "Ist auch das Wasser, das in der Quelle rinnt, allen eigen, wer wollce in Zweifel stellen, daß das Wasser im Krug nur dem gehört, der es geschöpft hat? “'6 Die mit dem modernen menschenrechclichen Common-Hcricage-of-Mankind-Prinzip begründetc Teilhabe-Forderung derjenigen Völker, die kcinen Krug besitzen, erkennt die Eigentumslegitimation durch Arbeitsleistung nicht mehr, oder jedenfalls nicht mehr in uneingeschränktem Maße an. Hierfür darf man, freilicls ein wenig spekulativ, eine doppelte, teils subjekrbezogene, teils objektbezogene Motivation vermuten. Die subjekrbezogene löst den strengen Zurechnungszusammenhang zwischen individueller Arbeitslcistung und technologisch potenziertem Arbeitsentag, ciner schier grenzenlos steigerbaren. Arbeitsproduktivitär zuf. Die Effizienz der modernen

12 UN Doc. A/CONF. 62/122. Unired Nawons Convention on the Law of the Sea, 10 Dectumber ig \&2, vgl. Riedel, a. a. O

13 J. Locke. Second 'Treause of Covverninent, V, 26.

14 J. J. Rousstax, Discours uber den Ursprung und die Grundlagen der Unglechlihet unter den Menschen, II.

is BVeriGE 69, 272, 100, st. Rspr.

$16 \mathrm{~J}$. Locke, Sccond Treatusc, V, 29 
Technik im jeweiligen Leistungszusammenhang kann weder einem (oder vielen) Einzelnen noch einem abgrenzbaren Kollektiv zugercchnet werden. Die objekrbezogene Motivation leugnet - mit wclcher Begründung auch immer - die unbegrenzte Aneignungsłăhigkeit der irdischen Güter. In dieser letzten Hinsicht berührt sich übrigens der moderne Common-Heritage-Gedanke wieder mit der Grund-Idee John Locke's, den man keinesfalls zum ideologischen Stammvater eines hemmungslosen, technikbasierten Ausbeutungskapitalismus zurechtstilisieren darf. Gott gab die Welt, sagt er, den Flcißigen und Verständigen zum Gebrauch und zu Eigentum, ${ }^{17}$ aber nicht unbegrenzt. "So viel, als ein jeder zu irgendwelchem Vorteil für sein Leben nutzen kann, bevor es verdirbt, darf er sich zu seinem Eigentum machen. Was darüber isc, ist mehr, als ihm zusteht, und gehört den anderen. ${ }^{18}$ Denen, die die Regenwälder Brasiliens oder dic Edelhölzer Afrikas schonungslos abholzen, sollte man die Lektüre (und Beherzigung) der Locke'schen Eigentumsphilosophie ebenso zur Pflicht machen wie den Verbrauchern auf der nördlichen Halbkugel, die die Nurznießer dieses Raubbaus sind.

III.

I. Zu den charakteristischen Antworten des Rechtssystems auf die Herausforderungen der Technik und ihrer Risiken gehört die zunchmende Austauschbarkeit von subjektivem und objektivem Recht. Aul der einen Scite fordert man die Ausdehnung des Kreises der Rechtssubjekte auf Tiere, Pflanzen, Flüsse oder Landschaften oder andere Gegenstände der unbelebren Natur. "Should trees have standing?" fragte Christopher D. Stone 1974, und die Zahl derer, die mit Ja antworten, wächst." Der allgegenwärtigen Bedrohung der Natur durch die Technik glaubr man nur durch ihre umfassende Subjcktivicrung begegnen zu können. Auf der anderen Seite neigt man dort, wo die Zuerkennung von subjektiven Rechtsansprüchen zu unabsehbaren Kostenbelastungen für den Staat führen könnte, dazu, ein subjekrives Grundrecht, wie z. B. "das Recht auf Leben und Gesundheir «, mit einem objekrivrechelichen Schutzmantel zu umkleiden. Der einzelne hat dann zwar nichr ohne weitcres ein einklagbares Recht auf unverseuchecs Trinkwasser, wohl abcr isc der Geseczgeber von Vcrfassungs wegen objekrivrecluclich verpflichter, ausreichende gesundheitsschützende Wasserrechisnormen zu erlassen. Eine inhaltliche Objektivierung ursprünglich rein individuell-subjekriver Grundrechtspositionen vollzieht sich auch als Folge der wissenschaftlichen sowie der technologisch-industriellen Arbeitsteilung. Dem Grundreche der Wissenschaftsfreiheit, Art. S Abs. 3 GG, entsprach die Vorstellung des über Inhalt, Methode und äußere Bedingungen seiner Forschung vollkommen frei entscheidenden einzelncn Wissenschaftlers. Daß dieses Bild die Realität moderncr Forschungszusammenhänge verfehlt, bedarf keiner Ausführung. Heute treten Rechte der kollegialen Mitbestimmung in gesetzlich oder institutionell geordneten Verfahren an die Stelle der autonomen Einzelentscheidung. Es ruäre jedoch ganz falsch, hierin nur einen Verlust an autonomer Wissen-

17 Derselbe, ebendort, V, 34

18 Derselbe, ebendorr. $V, 31$

19 Chrisiopher D. Stone, Umwek vor Gerncht. Dic Eigenrechte der Natur 1987 (engl. 1974); K. M. McyerAbich, Wegc zum Fneden mil der Natur, 1984; P. Saladin/J. Limbacher, Mensch und Natur: Hersusforderung fur die Rechespolizik, in: Menschengereche. Rechespolituscher Kongreß der SPD, Essen $19 \$ 6$, Arbeitsmaterialien. Kritusch und differenziert jeiza: H. Freiherr von Lersner Gibt es Eigenrechie der Natur', NVwZ 19\$8, g88. Vernesnend: VG Haniburg, B. v. 22.9. $1988, N V w Z 1988,1058$. 
schaftsfreiheir zu schen. Die Mitbcstimmung der an eincm überindividucllen Forschungsprojekt beteiligten Wisscnschaftler ist nicht Fremdbestimmung, sondern autonome Ausübung ihrer Wissenschaftsfreiheit. Auf der Ebene des produkciven, gesellschaltsrechtlich gebundenen Eigentums, etwa in ciner Aktiengesellschaft, spielt sich Entsprechendes ab. Moderne techniscle Leistungen sind nur durch den gcbündelten, hoch arbeitsteiligen Einsatz einer Vielzahl von Kapitalbcituägen und Arbeissleistungen zu verwirklichen. Wenn sich der Einzelne mit seincm Beitrag dem gemeinsamen Ziel und Plan unterordner, so muß man auch dies nicht als hereronomc, sondern als autonome Rechtsausübung verstehen. Entschcidende Bedeutung komm dann allerdings der rechtlicken Absicherung seiner verfahrensmäßigen Stellung und der mir ibr gegebenen Mitririkungschancen zu. ${ }^{20}$

2. Wenden wir uns nunmehr der Frage zu, welche Rechrsenrwicklungen auf der Ebene des inmerstaatlichen deurschen Rechts als unmittelbare Folge technologischer Veränderungen zu bcobachcen sind, so müssen wir zwei Linien unrerscheiden. Die erste zeichnee dic Herausbildung neucr, bishcr nicht bekannter, inhalelich bisher so nicht existierender Rechte nach. Die zweire betrifft die Fortentwicklung der bekannten Rechtsdogmarik, insbesondere der Grundrechtc; auch hier ergebcn sich im einzelnen zum Teil neue Rechtsansprüche aus aitcn, wohlbekannten Grundrechเеп.

Bei der Betrachtung der Rechte der ersten Kategorie ist man versucht, sie danach zu klassifizieren, ob sie Ausdruck eines optimistischen oder aber pessimistischen Technikbewußtseins sind. Odcr weniger gefühlsbetont: Wir können Rechte als Folgen erweiterer Naturbeherrschung durch die Technik von solchen Rechren uncerscheiden, die vor allem der Sicherung gegen die Gefahren der Technik dienen. Zu der ersten Gruppe, zu der international das Rechı auf das gemeinsame Menschheisserbe zählt, ist das Recht auf die sogenannte Grundversorgung durch den öffentlichrechtlichen Rundfunk in Deurschland zu zählen. Dieses ist zwar als Recht der öffentlichrechrlichen Rundfunkanstalten auf ungeschmälerte Aufgabenwahrnehmung gegenüber der privatrundfunkrechelichen Konkurrenz entwickelt worden." Dahinter stehr jedoch der auf das Sozialstaatsprinzip gegründece Anspruch des Einzelnen auf Tcilhabe an einem Minimum kulturelier Leistungen. Auch die moderne Konzeption des Rechres auf Bildung und auf Ausbildung, welche jedem Bildungswilligen grundsärzlich den Zugang zu der besten ihm möglichen Ausbildung eröffnet, ist hier zu erwähnen.

Weitaus zahlreicher sind die schon anerkannecn oder nur poseulierten Rechte, welche Schutz gegen die negariven Möglichkeiten moderncr Technik gewähren sollen. ${ }^{22}$ Das beginne mit der Wicderentdeckung des Grundrechts oder sogar Menschenrechts auf Sichcrheil, ${ }^{23}$ führt über das Grundrecht auf eine lebenswerte Umwelt, ${ }^{24}$ über das Recht auf Freiheit von Furcht vor technischon Gefahren"1 sowie vor staatlicher Überwachung ${ }^{26}$ bis zu dem daran anknüpfenden Grundrecht auf

20 Zur (Grundreches-)Freihell durch und in Minbestummung vgl. AX-GG-Denninger, vor Art. i Rdne. $18 f$. Dort auch Nachweis abw. Meinungen.

21 BVeriGE 73, $118,157$.

21 Das wird besonders deutlich an der „Erklarung der Reche künfuger Genemuonen ", welche P. Saladin und C. A. Zenger formulien haben. Vgl. diesclben. Ruchte künfuger Generauonen, Basel/Frankfurd/M. 1988, S. 466 . Don auch, S. 67 ff, zum Common Herisage of Mankind-Ansaiz.

2) G. Robbers, Sicherhen als Menschenrecht, 1987; J. Isensee, Das Grundrechi auf Sicherheit. Zu den Schuizpolichren des freiherelichen Veríassungssizates, $19^{8} 3$.

24 Nachweise zum Stand der Diskussion $\left(b_{15} 198_{3}\right)$ in: Staxtszielbestummungen/Gesecrgebungsaufrrage. Bcriche der Sachverständigenkommission, hrsg. v. BMin des Innern, BMin der Justız, 1983, Rdnr. $13 i-1$ to.

as H. Hoimann, Rechtsfragen der alomaren Fntsorgung, $198 \mathrm{l}$, j08 C.

$26 \mathrm{Vgl}$. Denninger, Verfassungserctue und Schulx der Verlassung, VVDSiRL Hefr 3\%. 1979, $26 \mathrm{ll}$. 
informationelle Sclbstbestimmung und zu dem Recht cines Menschen auf Kenntnis seiner biologischen Abstammung als Schutz gegen Experimente mit heterologer in vitro-Fertilisation. ${ }^{27}$ Es kann nicht verwundern, daß ein erheblicher Teil diescr Rechte, wic das Rechs auf Freiheit von Furcht, die informationellc Sclbstbestimmung als Ausdruck des allgemeinen Persönlichkeitsrechis ${ }^{28}$ oder auch die Begrenzung der humangenetischen Experimente rechtsdogmatisch unmitcelbar am Menschenwürdesatz des Art. I Abs. I Grundgeserz festgemache werden müssen. Denn der Zugriff der Technik erfolgt in diesen Fällen unmittelbar auf die Identitär der menschlichen Person. Bei der anonymen heterologen Befruchtung soll dem entstehenden Lebcwesen ein Teil seiner biologischen Identität gewissermaßen unterschlagen werden. Bei der vom Individuum nicht mehr kontrollierten oder kontrollierbaren. Fremdverfügung über persönliche Daten wird die Herausbildung der sozialen Identitär des Menschen partiell oder gänzlich zerstörr. In beiden Fällen ist das Individuum nur noch Objekt, nicht aber Subjekı seiner Um- und Mitweltbeziehungen. Die unvermeidliche unmitrelbare Rückführung der Rechrsposicion des Einzelnen auf den Menschenwürdesacz bedeuter freilich zugleich, daß die rechtliche Diskussion darüber niche ohne Rückgriff auf philosophisch anthropologische Prämissen und mithin nicht ideologiefrei geführt werden kann. Es isc aber jedenfalls menschlicher, diese Auseinanderserzung zu leisten als sich blindlings allen technischen Machbarkeiten auszuliefern.

3. Die zweite der vorhin angedeutcten Rechtsentwicklungen verläuft weniger spektakulär, wcil sic jedenfalls terminologisch darauf verzichtet, neue Rechte zu präsentieren, sondern vielmehr Inhalt und Funktion bestchender Rechte umbildet. Sie setzt sich aus einer Vielzahl oft nur richterrechelich enrwickelter Rechrsänderungen zusammen; und es ist nicht ganz cinfach, deren gemeinsamen Nenner begrifflich präzise zu bestimmen. Ich möchte dies dennoch versuchen, indem ich sechs Beispiele zur Diskussion stelle:

a) Dem Idealbild rechtsstaatlicher Bcstimmtheit von Eingriffsgesetzen entspräche es, wenn die Sphäre des Bürgers und die Ingerenzmögliclikeir des Staates durch feste, möglichst zahlenmäßig bestimmte Werte voneinander geschieden wären. ${ }^{29}$ Der Bürger wüßte dann, worauf er sich einzurichten hat, die Handlungen des Staates wären für ilın kalkulierbar. Für das Recht der technischen Sicherheit ist dieses Modell überholt. Dic sich rasch verändernden technischen Risiken und Gcfahren und die noch rascher voranschreitende Erkenntnis derselben, ${ }^{\circ}$ die keineswegs immer von ebenso raschen Fortschritren in der entsprechenden Schurz- und Vorsorgetechnik begleitet ist, haben die Rechuprechung des Bundesverfassungsgerichts veranlaßt, dem Gesetzgeber nicht mchr die Statuierung fester Grenzwerte, sondern nur noch die Beachtung des Grundsatzes bestmöglicher Gefalırenabwehr und Risikovorsorge aufzugeben." Dem genügt der Gesetzgeber, wenn er beispiels-

27 Vg). H.B.Schlegel (Hrsg.), Gentechnologie und In-vitro-Ferilisation, Kolloquium der Akademie der Wissenschatien ze Götungen, 23. Januar 1987, 1988. Dort vor allem die Bcitráge van C. Starck und G. Patzig; fernct $\Lambda$. Eser. Gentechnologic und Reche: Der Mensch als Objeke von Forschung und Tecbrik, in: Menschengereche (Fn. 19), 1986, isff. Zum Grundreche au Kennenis der eigenen Abstammung neuestens: BVeriG, Urtel vom $31.1 .1989, J 2$ 1989, $335 \mathrm{~m}$. Anm. v. C. Starck.

18 So deuelich BVerfGE 6s, 1, 41: Arı. 2 Abs. I GG in Verbindung mir An. I Abs. I GG. Zum allgemeunen Persönlichketrsrecht An. 2 Abs, I GG vil. bes. v. MangoldU/Kleın/Starck, GG, 3. Auf. 1981, Art. 2، Rdnr. 64 ff, neuestens BVeriG, U. V. 31. 1. 1989, JZ 1989, $33^{6}$.

29 Zum "Niedergang* des rechtsstaaclichen Rechussicherherts-ldeals insgesame vgl. E. Dennınger, Der Präventions-Staat, KJ 1988, 1.

30 Die jurisusche und naturwissenschaficliche Diskussion uber "Grenzwerce gibı davon Zeugnis. Vgl. nur G. Winter (Hrsg.), Grenzwerte, 1986 (Umwcltrechtiche Studien Band I, hrsg. von Batus/Rehbinder/ Wincer); F. Nicklisch (Hrsg.), Prävention im Unwelteccht. Risikovorsorge, Grenzwerte, Haftung, 1988. 3. BVcricE $49,89,139$ (Kalkar); \$3, 30, 88 (Mülhesm-Kärlich). 
weisc im Atomrecht dem Anlagenbecreiber vorschreibc, daß cr dic *nach dem Stand von Wissenschaft und Technik « erforderliche Risikovorsorge zu treffen habe $(\$ 7$ Abs. 1 AcomG). Entsprechend wird bei der Begrenzung von Lärm- oder Schadstoffimmissioncn verfahren.." Dieses sogenannce Prinzip eines »dynamischen Grundrechusschurzesu's hat einen Vorteil, aber mehrere Nachteile. Der - theorecische Vorteil für den Bürger liegt in der Zusicherung optimalen Grundrechrsscluutzes; die Nachteile liegen in der Entmachtung der Legislative und in einem schwer kontrollierbaren Machtzuwachs für die Exekutive, ferner in der permanencen Umstrittenheit der tatsächlich praktizierten Grenzwerte, was zu langwierigen und uferlosen Prozessen führt. An die Stelle klarer demokratisch legitimierter, sachlich eindcutiger Entscheidungen tritt ein undurchsichtiges Geflecht aus sachverständiger Beratung, interessengebundener Einflußnahme und administrativem Politikersatz. Dic Theorie (des Bundesverfassungsgerichts) isc löblich, gibe sie doch dem Geserzgeber sogar auf, scine Gesetze je nach besserer wissenschaftlicher Einsicht "nachzubessern «, doch die Praxis ist kümmerlich; sic hinkt nicht nur den jeweils einschlägigen Katastrophen (von der Nordseeverseuchung bis zum Ozonloch) hinterher, sondern sic tur dies auch noch im Schneckentempo internationaler Harmonisierungskonferenzen.

b) Dem Idealbild rechesstaaclicher Ordnungsverwaltung entspricht dic sogenannte gebundene Erlaubnis. Der Bürger soll einen Rechrsansprucl auf die Erteilung der Verwaltungsgenehmigung haben, wenn er die gesetzlich möglichst genau umschriebenen Vorausserzungen der Genehmigungserceilung erfüllt. Denn der Staat will die Aktivität des Bürgers, z. B. dic Ausübung eines Gewerbes, ja nicht vcrbicten, sondern nur im Interesse des Gemeinwohls präventiv kontrollieren. Auch diescs Prinzip fälltr im Bereich besonders gefährlicher Technologien dahin. Die Verwaltung nimme cin nichr näher definieres Versagungsermessen in Anspruch, also die Kompetenz, die erforderliche Erlaubnis zu versagen, obwohl der Bürger alle von Gesetzes wegen geforderten Bedingungen erfüllt har. ${ }^{34}$ An die Stelle einer dem Staat gegenüber zweifelsfrei durchsetzbaren Grundrechrsausübung tritt eine Risiko- und Chancengemeinschaft zwischen Bürger und Staat nach Maßgabe eines mit vielen Ungewißheiten behafteten Risikokalküls.

c) Führen die Bedingungen moderner Technik im Wrirschaftsrecht vor allem zu Unschärfen der Rechtsinhaltc und zu Unsicherheiten der Rechtsanwendung, so bewirken sie in anderen Rechrsgebieten, etwa im Polizei- oder Umweltrecht, eine Aufösung der Subjektkategorie. Dem rechtsstaatlichen Idcal des Polizeirechts entspricht es, daß polizciliche Maßnahmen nur gegen den Störer gerichcet werden können, d.h. nur gegen denjenigen, der selbst eine politische Gefahr verursacht hat." Infolge der technischen Entwicklung wird dieser Grundsatz in zweierlei Hinsichr rclativiert: Zum einen wird die individuelle Zurechnung einer polizeilichon Gefahrenverursachung dort fragwürdig, wo zahllose Einzelne in nicht meßbaren Einzelbeiträgen zur Entstehung einer Gefahrenlage zusammenwirken (Luftverschmutzung, Waldscerben, Grundwasser- und Bodenverseuchung). Zum anderen sind mehrere moderne polizeiliche Übcrwachungs- und Erforschungstcchniken (Videoüberwachung, elektronische Dateien oder Rasterfahndung) von ihrer Technik her so angelegt, daß sie Unbeteiligte ebenso wie Beteiligte oder nur potentiell

12 Vgl. etwa $\$$ 7a WHG: ,allgemein anerkannte Regeln der Technik * und strenger aSuand der Technik * als Maßstäbc. Zur Lämabwehr vgl. BVeriGE $56,54,78$ ff. (Fluglärmbekämpfung).

3) BVerfGE 49, 89, 137: "Nachbesserungspflichi * das Geseizgebers: BVerfGE 56, 54, 79.

14 Excmplariscli im Aromrecht. Vgl. (mit xahlreichen Nachiv.) BVeriGE 49. 89, $144 \mathrm{ff.}$

is Vgl. statt vieler: Drews/Wacke/Vogel/Martens, Gefalırenabwehr, 9. Aufl. 1986, 290ff., V. Görz, Allgemeines Polizel- und Ordnungsrechr. 9. Aufl. 1988. Rdnr. 18 s ff. 
Beteiligte effassen. Das heißt, polizciliches Handeln richtet sich notwendig nicht mehr nur gegen den oder die Störer, sondern auch gegen völlig nharmlose Bürger. ${ }^{6}$ Im Umweltrecht tritr an dic Stclle der liberal-rcchtsstaatlichen „Störermehrheiru eine Solidarhaftung aller Benutzer in Gestalt einer Gefährdungshaftung (z. B. im Wasserrecht). ${ }^{37}$

d) Dieser Ausweitung des Kreises der Pflichtsubjekte entspricht u. U. eine solche des Kreises der Begünstigten. Nach dem Vorbild der Entwicklung im Baurecht hat das Bundesverwaltungsgericht jüngst den Nachbarschutz auch im Wasserrecht anerkannt. ${ }^{8}$ Während die Gerichte hier und anderswo unendlich mühsam versuchen, durch cine Vielzahl einzelner zweipoliger (Staar/Bürger) Entscheidungsverfahren cine Art flächendeckender patchwork-Gerechrigkeit zusammenzubasteln, würden die technischen Bedingungen, etwa für cinc gemeinverrägliche Abwasscreinleitung, ein viclpoliges Zuteilungs- und Haftungsübernahmeverfahren erfordern, an dem sämtliche Beteiligten mitzuwirken hätten.

e) Damic begegnen wir erneut der Interdependenz von Rechtsinhalt und Verfahrensgestaltung zur Rechtsơurchserzung. Diese dem Juristen seir dem römischen Aktionensystem an sich vertraute Erscheinung erfährt durch die technologische Entwicklung eine charakteriscische Ausprägung. Während nach dem Idealbild rechtsstaatlichen Verfahrensrechts ein inhaltlich definiertes Recht je nach der Tatsachenlage und ihrer Beweisbarkeit entweder garzz oder teilweise zuerkannt wird oder nicht, wird bei komplizierten technikrechtichen Sachverhalten der Rechtsinhalt selbst nach Maßgabe des Forgangs des Verfahrens veränder. Dabei kommt dem Faktor Zeit cin um so größeres Gewicht zu, je stärker er auch die technische Entwicklung auf dem betreffenden Gebict und damic die technische Obsoleszenz beherrscht. Ein Bcispiel aus dem Arzneimitrelrecht mag dies illustricren:39 Obwohl der Gesetzgeber bindend vorschreibt, daß die Gesundheitsbehörde die Entscheidung über dic Zulassung cines neuen Arzneimittels zum Marks innerhalb von vier, äußersienfalls sieben Monaten zu rreffen hat, führt die Kompliziertheir der toxikologischen und pharmakologischen Prüfungen durch das Gesundheitsamt dazu, daß die Ancragsteller mehrere Jahre auf den Zulassungsbescheid warcen müssen. Der Zeitverlust entwertet nicht nur einen evtl. gleichzeitig beantragten Patentschutz, sondern weitgehend auch den nach Dutzenden von Millionen Mark zählenden Investicionsaufwand für die Encwicklung des neuen Präparates. Selbst wenn man einräumt, daß die getroffenen Fristregelungen nicht nur als objekrives Recht für die Behörde verbindlich sind, sondem auch Grundlage für subjekrive Klageansprüche der Arzneimitcelhersteller sein können, so ist doch nicht ohne weiteres zu sehen, wie sie rasch zu ihrem Recht kommen können. Denn auch das Gericht könnte seine Entscheidung nicht treffen, ohne sich zuvor msachkundig* gemacht zu haben. Und dies eben dauert offenbar Jahre. Der Rechrsstaat stößt hier an rechnologische Grenzen seiner Verwirklichung.

1) Dieses und andere Beispiele belegen dic Richrigkeit der Feststellung der Verfassungsrichcer Simon und Heußner, daß nur durch eine geeignete Gestaltung des Verfahrensrechts verhindert werden kann, daß der Bereich zwischen Recht und

$36 \mathrm{Vgl}$. M. Knicscl/H. 'legemeyer/J. Vahle. Handbuch des Datenschutzex fur Sichcrheresbehórden, 1986, zu den Techniken: \$. 11611 , 226 II.: zur rechilichen Zulässıgkete: S. 249 If. Ferner: U. Kauß, Der suspendiere Datenschutz bes Polizcı und Geheımdiensten, igS9.

37 Bespicl: $\$ 22$ WHG.

3 BVerwGE 78,40 mil Verwisung aul BVerwGE $\{2.122,129$ fl. st. Rspr. (Baurccht)

39 S. \$27 Abs. I AMG, gemäß EG-Richulinic 65/6, EWG, Art. 7, v. 26. 1. 1965. Vgl. dazu E. Dunninger, Der *Rechrsscaata im (Anuags-)Stau, Pharm. Ind. 51 (1989), 249ff. 
Technik zu einem juristischen Niemandsland wird..$^{\circ}$ Dabei sind die Verfahren so anzulegen, daß die cinander widerstreitenden Interessen der Betroffenen, die Belange des Gemeinwohls und die technologisch geforderte Sachrichtigkeit zu einem vernünftigen Ausgleich gebracht werden können. Was hierzu im einzelnen erforderlich ist, bedarf erst noch der Erforschung." Bei alledem wäre es jedoch falsch, die optimale Verwirklichung von Vcrfahrensgerechrigkeit allein schon für eine ausrejchende Bedingung einer auch in der Sache selbst richeigen Entscheidung zu halren. Ware dies anders, so könnce man sich den schwierigen Prozeß einer politischen dernokratischen Entscheidungsfindung crsparen und sich mit einem Zusammenwirken wissenschaftlich-technischer Sachverständiger mit unabhängigen Richtern begnügen.12 Fragz man nun, nach welchen materiellen Kriterien die Gerichte technikrechuliche Kollisionslagen entscheiden, so szößt man letztlich immer wieder auf den Grundsatz der Verhältnismäßigkeit. Er trite alierdings vielfach auch in der Verkleidung schcinbar subjektbezogener Kategorien wie Zumutbarkeit, Rücksichtnahmcgebor, Schwere und Unerträglichkeit cines Eingriffs auf. In Wahrheit handelt es sich dabei um richterliche Verlegenheirsformeln, welche den Versuch einer objektiver Maßstabsfindung mit Hilfe appelativer, das subjektive rechtsgefühl ansprechender Kacegorien wagen. Die Inkommensurabilitäı zwischen der Präzision der Zahlensprache des Naturwissenschafticrs und Technikers und der bewertenden Sprache des "Gerechrigkeitskünstlers « (des Juristen) wird nirgends deutlicher als hier."

4. Was also ist das Neue an den neuen Rechten im technologischen Zeitaiter? Vielleicht die Einsicht, daß die uralten Schwierigkciten der Menschheit mit der Gerechtigkeitauch durch den wissenschaftlich-technischen Fortschritt nicht gelöst werden. Erwächst daraus das Bewußtsein, sich ihnen immer wieder verantwortlich stellen zu müssen, so ist das schon viel.

40 BVerfGE 33, 30, 76 (Abry. Voum). Der Jubilar, dem diese Zeilen gewidmes sind, hat die oProzeduralisienung" von Rechs fruhzeiug und wsederholt thematister, Vgl. nur R. Wiethölter, Matenalization and Procedumlization in Modern Law. (1982), Veróff. in: G. Tcubner (Hrsz.). Dilemmas of Law in the Wellane Siste $(1996), 221$ ff.

11 Daxu demnachse: E. Denninger, Recheliche Grenzen der Mitwırkung außerstaadicher Einrichtungen bei staablichen Normscizungs- und Kontrollverfahren, bes. 3. Téil, II, 4.

42 In diese Richtung tendieren Vorschliage wie der von H.-J. Papicr, Der Vorbchale des Gescizes und seiner Grenzen, in: Gotz/klesn/Starck (Hrsg.). Die offertiche Verwa)tung zwischen Gereuzgebung und richecrlicher Kontrolle, $1985,3611.66 \%$.

43 Dazu E. Denninger. W/ssenschaftlich-technischer Sacliverstand und staaliche Technologiesteuerung uurch Recht, in: Festschrife für ]. Habermas, igs9. 